\title{
Current Situation and Analysis of the Medical Physics Experiment in Local Medical College
}

\author{
Jia Guo ${ }^{1, a}$, Weiwei Jiang ${ }^{2, b}$ and Benchao Zhu, c \\ ${ }^{1}$ Hubei University of Medicine, Mathematics and Physics Department, Shiyan 442000, China \\ ${ }^{2}$ Hubei Dongfeng Automobile Technician College, Shiyan 442000, China \\ a416355087@qq.com, bjww0507@163.comn, ‘31430166@qq.com
}

Keywords: Local medical colleges; Current situation; Medical physics experiment; Course analysis; Education reformation

\begin{abstract}
In this paper, the comparative analysis method is used to compare the relation between the key comprehensive university and local medical college, medical physics experiment course. The results show that there are many factors, in the subjective and objective, cause the same one course having the different form and the effect of the learning. Then, we discuss these factors in detail from many aspects. Combined with the current policy of promoting the transformation of local colleges by china education department in 2014 , this paper also discusses how to integrate the application of the type, skills of the "medical physics experiment" course teaching reform. Throughout this paper, we can find that the actual situation of medical physics experiment course is very bad, but, under the national new policy of transformation, the reform of medical physics experiment teaching in local medical college has encountered an unprecedented good opportunity.
\end{abstract}

\section{地方医学院校《医学物理学实验》课程现状及 分析}

\author{
郭佳 ${ }^{1, a}$, 蒋薇薇 ${ }^{2, b}$, 朱本超 ${ }^{1,}$ \\ 1. 湖北医药学院, 数理教研室, 中国 湖北十堰 442000 \\ 2. 湖北东风汽车技师学院, 中国 湖北 十堰 442000

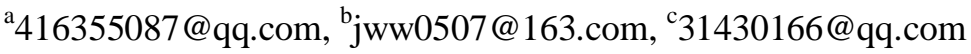

摘要: 本文采用对比分析法, 将重点综合性大学和地方医学院校《医学物理学实验》 课程进行了横向和纵向对比。对比的结果显示, 在主观上、客观上都有着许多因素造 成了同一门课程开设形式、开设效果的巨大差异, 并对这些因素进行了细致分析。结 合当下教育部提倡地方高等院校转型政策，本文还探讨了如何接轨应用型、技能型的 《医学物理学实验》课程教学改革。通过本文, 将会发现, 当下地方医学院校《医学 物理学实验》课程现状堪忧, 但是在国家新的转型政策刺激下, 地方医学院校《医学 物理学实验》课程教学改革遇到了前所未有的好机遇。

关键词: 地方医学院校; 医学物理学实验; 课程现状; 课程分析; 教学改革

\section{1. 引言}

目前, 在我国各类医学院校开设的必修类基础课程《医学物理学实验》, 是《医学物理 学》课程理论教学的配套教学内容。该课程的开设必要性可以从两方面简单论证, 第 一, 物理学作为实验性科学, 所有的理论必须经过实验验证才能被认可和接收学习。 因此, 作为大学物理在医学院校的具体应用, 《医学物理学》必须要开设配套的实验教 
学。第二, 从医学院校特点来讲, 医学院毕业生从事医疗相关工作, 大部分都是操作 性非常强的, 因此在所有非人文类学科学习中, 对于医学生的技能有较高要求。这也 是为何, 在 2014 年 2 月国务院和教育部做出鼓励地方本科院校转型发展 ${ }^{[1,2]}$ 为技能型、 应用型高校时, 地方医学院校作为首批试点院校的原因。既然地方医学院校转型势在 必行, 那么作为服务性学科的《医学物理学实验》在课程教学上, 必须要经过一些改 进才能更好地适应地方医学院校的功能转换。在完全梳理《医学物理学实验》课程教 学改革之前, 本文认为十分有必要对地方医学院校《医学物理学实验》课程开设现状 及其中弊端进行分析。本文首先对我国目前医学院校物理实验课程现状进行整体分析, 然后对地方医学院校物理课程进行详尽介绍, 特别是以笔者所在学校为例。最后, 对 结合应用型和技能型培养目标，医学物理学实验课程可能进行的改革进行前瞻性梳理。

\section{2. 医学院校《医学物理学实验》课程开设现状对比研究}

《医学物理》与《医学物理实验》属于交叉学科, 是应用物理学的一个分支, 是研究把 物理学理论及物理学技术应用于医学诊断, 医学治疗及疾病预防的学科领域。在所有医 学类院校中, 往往是一年级学生必修课程。但是在不同层次, 不同地域的医学院校, 其开设现状是参差不齐的。在重点综合性大学, 如北京大学、清华大学、复旦大学、 浙江大学等, 将医学物理实验作为大学物理实验的一种, 结合这些学校强大的财政、 师资等优势, 医学物理学实验项目的开发和实验课程上课形式都非常科学。以复旦大 学的精品课程, 《医学物理学实验》为例 $[3,4]$, 复旦大学公布的医学物理学实验教学 组有 6 名专职教师, 承担全校医学生的物理实验教学, 下表是该校 2016 年开设的物理 实验项目

表 1 复旦大学医学物理学实验项目设置

\begin{tabular}{|l|l|}
\hline 实验 1 弯曲法测人造骨杨氏模量 & 实验 2 毛细管法测液体黏度 \\
\hline 实验 3 眼睛的光学原理及物理矫正 & 实验 4 压力传感器特性及血压, 心率测量 \\
\hline 实验 5 人耳听阈、痛阈曲线研究 & 实验 6 心电图机技术指标的测量及使用 \\
\hline 实验 $7 \mathrm{~A}$ 型超声探测及 B 型超声成像原理 & 实验 8 X 射线特性研究及透视 $\backslash X-C T$ \\
\hline 实验 9 核磁共振基础及成像原理 & 实验 10 设计性研究性实验 \\
\hline
\end{tabular}

其中实验 10 设计性研究性实验项目的目的是训练学生物理与医学知识的交叉运用能 力、动手能力、研究能力、团队协作能力及创新意识。可选范围很广, 例如: 医用监 护仪模拟组装、肺呼吸模型实验装置研制、肺呼吸顺应性曲线实验设计、脉搏计数器 组装、家用自主听阈曲线测量装置研究、心电图波谱分析、 $\mathrm{A}$ 超测量杨氏模量及验证声 反射规律、血栓与血压关系的模拟研究等。作为必修的《医学物理实验》共 36 学时, 9 至 10 个实验项目, 每个实验项目 4 学时, 每个实验台 1 到 2 人操作仪器, 自主预约 制度。

作为对比, 在表 2 中列出了笔者所在医学院校, 湖北医药学院（湖北十堰）医学临床 专业开设的医学物理实验项目及基本信息

\section{表 2 湖北医药学院医学物理学实验项目设置}

\begin{tabular}{|l|l|}
\hline 实验 1 拉伸法测量钢丝杨氏模量 & 实验 2 奥氏粘度计测量酒精粘度系数 \\
\hline 实验 3 金属导体的温差电现象 & 实验 4 衍射法测量光波波长 \\
\hline 实验 5 显微镜放大倍数的测量 & 实验 6 基本电子仪器的使用 (万用表 $)$ \\
\hline
\end{tabular}

本校只有一名专职实验教学的教辅 1 人, 兼职教师 3 名。实验总共学时 18 , 共开设 6 个实验项目, 每个实验项目 3 学时, 每个实验台四到五人, 不能预约。作为实验室建 
设, 湖医药物理实验室还拥有以下几个实验项目可选: 人耳听阈、痛阈曲线研究; 变 温法测量液体粘度; 基本电子仪器的使用 (示波器); 温度传感器箱。

\section{3. 地方医学院校《医学物理学实验》课程的主要问题}

通过上表 1 和表 2 及其课程说明, 我们可以清晰看到地方医学院校相对重点大学医学 物理学实验的主要差距表现在以下几个方面:

表 3: 地方医学院校和综合性大学《医学物理学实验》差异对比

\begin{tabular}{|c|c|c|}
\hline 对比项目 & 地方医学院校 & 重点综合性大学 \\
\hline 实验专职师资 & 较少 & 较充足 \\
\hline 实验项目数量 & 较少 & 充足 \\
\hline 实验项目类别 & 经典物理 & 经典物理+近现代物理 \\
\hline 实验仪器操作人数 & $4-5$ 人/台 & $1-2$ 人/台 \\
\hline 实验课程选课形式 & 无法自选安排时间 & 自选安排时间 \\
\hline
\end{tabular}

造成地方医学院校《医学物理学实验》课程的主要问题, 有着客观和主观方面的因素。 这些问题主要表现在:

客观上，地方医学院校受到体质，财政的影响非常大，特别是一些内地地方医学院校， 在上级管理部门的统筹安排下，对实验仪器的购置、报废等手续相对僵化且效率偏低。 这些造成了物理类实验仪器的添置在地方医学院校显得比较繁琐, 客观上造成了实验 项目相对落后 [5]。

主观上, 地方医学院校的领导, 对公共类基础课程的理解不科学, 认为地方医学院校 是服务地方经济, 不需要学习太多非医学相关知识。人为地造成医学物理实验课程教 学学时, 教学师资等因素落后。在物理理论学时无法保证相关内容讲解的前提下, 直 接开设近现代相关的医学物理实验是无法完成的。另外, 地方医学院校对实验课程自 选形式的不重视，也造成了实验课程僵硬缺乏科学地利用率 [6]。

学生层次上, 地方医学院校招收的学生层次相对于重点综合性大学也有不可忽略的差 距。这就造成了很多时候, 地方医学院校开设《医学物理学实验》课程时, 需要考虑 到学生对实验仪器人为的破坏性, 因此往往会开设一些耐用性很高的实验项目, 而这 些实验项目更多地集中在经典物理学 $[7]$ 。

这些因素的综合作用结果就是, 地方医学院校《医学物理学实验》课程现状堪忧, 开 设内容不科学, 师资欠缺, 仪器购置效率低下, 学生反应极其乏味, 恶性循环地造成 主管领导更不重视医学物理实验。

\section{4. 地方医学院校《医学物理学实验》课程与应用型、技能型接轨的改革}

在地方医学院校转型势在必行的前提下, 《医学物理学实验》课程的如何更好地与应用 型和技能型医学生接轨, 是每个从业人员必须面对的。因此, 在如此强大的外因作用 下, 地方医学院校课程改革遇到了一个好的机遇。对《医学物理学实验》课程而言, 做好应用型、技能型培养模式的接轨，主要做到以下几点:

第一, 地方医学院校应该借此转型机遇, 引入科学的选课制度, 将学生自主性选课机 制引入。这样学生可以在一年级或者二年级结合医学相关知识的学习, 更好地操作医 学物理实验。同时还有利于学生主观能动性的调动 $[8]$ 。

第二, 地方医学院校应该借此转型机遇, 将医学物理学和医学物理实验进行一次综合 性科学调研和改革, 对授课内容, 实验项目, 理论学时的删减及实验项目的增减进行 细致地调查。因地制宜地给出符合本校发展模式的物理教学形式 [9]。

第三, 地方医学院校应该借此转型机遇, 将附属医院的近现代物理相关医学仪器进行 
重新梳理, 彻底改进医学物理实验项目滞后等现状。只有跟学生今后技能相关地实验 项目才能真正为学生所关注和有兴趣 $[10]$ 。

第四, 地方医学院校应该借此转型机遇, 将医学物理实验设计性实验同重点综合性大 学进行接轨。重点综合大学开设的设计性应用型医学物理实验, 从本质上就是结合临 床技能和应用的相关知识。但是这种转变需要学校相关领导科学对待物理实验的功能, 需要政策和财政的高效支持。

\section{5. 结论}

本文通过地方医学院校和综合性重点大学《医学物理学实验》课程实验项目的对比, 在主观上、客观上都有着许多因素造成了同一门课程开设形式、开设效果的巨大差异， 并对这些因素进行了细致分析。结合当下教育部提倡地方高等院校转型政策, 本文还 探讨了如何接轨应用型、技能型的《医学物理学实验》课程教学改革。通过本文, 将 会发现, 当下地方医学院校《医学物理学实验》课程现状堪忧, 但是在国家新的转型 政策刺激下, 地方医学院校《医学物理学实验》课程教学改革遇到了前所未有的好机 遇。

\section{References}

[1] Dong HL. Right time to transformation of local undergraduate colleges (Education vision-focusing on the transformation of undergraduate colleges (I)) [N], People's daily, May 8, 2014, eighteenth edition: Education weekly.

[2] Dong HL. How to transformation of local undergraduate colleges (Education vision-focusing on the transformation of undergraduate colleges (II)) [N], People's daily, May 8, 2014, eighteenth edition: Education weekly.

[3] Ji Min. Medical Physics Experiment [M], Bei Jing: People's Health Publishing company, 2009.

[4] Fudan Medical Physics Teaching Lab, web site: http://phylab.fudan.edu.cn/doku.php?id=course:medical

[5] Song Qinghuan, Discussion on the teaching of medical physics experiments in Medical College [J], Chinese Modern distance education of traditional Chinese medicine, 2015(8): 119-120.

[6] Li Chaoyang, Reconstruction of teaching contents and reform of teaching mode in medical physics experiment [J], Journal of Mathematical Medicine, 2016(1):154-156.

[7] Tang Xiaonian, Zhang Fengqin, Tang Xiao, Zhu Ming, Problems and Reform in experimental teaching of Medical Physics [J], Chinese Northwest Medical Education, 2015(2):324-327.

[8] Cui Chunyu, Mo Zeng, Liao Xinhua, Discussion on the design of Medical Physics Experiment[J], Modern medical and health, 2016(4):601-602.

[9] Zhang Zhiwei, Discussion on the experimental project of medical physics with specialty [J], Science and technology vision, 2015 (26): 189-189.

[10]High Qinghe, crystalon, Wang Heyu, Liu Haiying, On the teaching reform of medical physics experiment [J], Continuing medical education, 2015 (7): 15-16.

作者简介: 郭佳 (1986 年一), 男, 湖北十堰, 助理实验师, 主要从事医学物理学实

验 E-mail: 416355087@qq.com 